Mon. Not. R. Astron. Soc. 000, 000-000 (0000) Printed 16 October $2018 \quad$ (MN LATEX style file v1.4)

\title{
A simple tool for assessing the completeness in apparent magnitude of magnitude-redshift samples
}

\author{
Stéphane Rauzy \\ Department of Physics and Astronomy, University of Glasgow, Glasgow, G12 8QQ, UK.
}

Accepted . Received ; in original form

\begin{abstract}
A new tool is proposed for finding out the completeness limit in apparent magnitude of a magnitude-redshift sample. The technique, closely related to the statistical test proposed by Efron \& Petrosian (1992), presents a real improvement compared to standard completeness tests. Namely, no a priori assumptions are required concerning the redshift space distribution of the sources. It means in particular that neither the clustering nor the evolution of the mean number density of the galaxies do affect the result of the search.
\end{abstract}

Key words: cosmology: large-scale structure of Universe - galaxies: distances and redshifts, luminosity function - methods: statistical, data analysis - astronomical data bases: miscellaneous

\section{INTRODUCTION}

Extracting the intrinsic characteristics of the galaxies population from magnitude-redshift samples (e.g. the luminosity function, the power spectrum of the spatial density fluctua' tions) remains one of the major concerns of observational cosmology. The task is somehow complicated due to the presence of selection effects in observation, e.g. a detection threshold in apparent fluxes. Because a part of the population is indeed not observed, standard statistical methods lead in general to biased estimate of the genuine parameters characterizing the population. While correcting for such biases is hardly feasible when intricate selection effects are at work, the problem has been fortunately handled in some special cases.

Samples complete in apparent magnitude obviously deserve mention. Indeed, magnitude-redshift data truncated to a lower flux limit has been extensively studied in the literature. Powerful methods have been developed in such a case for fitting or reconstructing the luminosity function of the galaxies population (for example the $C^{-}$method of Lynden-Bell 1976, the maximum likelihood fitting technique of Sandage et al. 1979). If the sample is furthermore complete in redshift, sophisticated methods have been proposed for estimating the power spectrum of the galaxies distribution (e.g. Heavens \& Taylor 1997) and for inferring the cosmic velocity field from magnitude-redshift data (e.g. Rauzy \& Hendry 2000, Branchini et al. 1999).

Flux limited magnitude-redshift samples can be built by primarily selecting from a parent magnitude sample all the objects brighter than the adopted flux limit. In a sec- ond step is collected the redshift of each of these sources (in this case the sample is complete in redshift), or a random subselection of these galaxies. By following such a strategy of observation, the completeness in apparent magnitude is essentially warranted as long as the parent sample is itself complete up to the flux limit. However, the completeness of the parent sample is in general difficult to assess. Undesired selection effects in observation are often at work and to some extent, the selection process depends on how are defined and measured the magnitudes of the galaxies (e.g. isophotal, visual, total magnitudes), how surface brightness threshold affects the sample, etc... (see for example Sandage \& Perelmuter 1990, Petrosian 1976, Driver 1999). Moreover, the parent sample may have been selected using criteria involving other observables not straightforwardly related to magnitudes (e.g. diameters, fluxes in a different passband). It turns out that the completeness assumption, a crucial prerequisite for applying any fitting and recontruction methods mentioned above, must be checked thoroughly.

A classical test for completeness in apparent magnitude is to analyse the variation of the galaxies number counts in function of the limiting apparent magnitude (Hubble 1926). This test, which presupposes that the galaxies population does not evolve with time and is homogeneously distributed in space, is however not very efficient. It is difficult to decide in pratice whether the deviation of the counts law is due to the presence of clustering and evolution of the galaxies luminosity function, or is indeed created by incompleteness in apparent magnitude. Including the redshift information, the $V / V_{\max }$ test of Schimdt (1968) has been also used for assessing the completeness of magnitude-redshift samples (see 
for example Hudson \& Lynden-Bell 1991), but suffers unfortunately from the same major drawback than the Hubble completeness test.

Efron \& Petrosian (1992) have thoroughly analysed the statistical properties of magnitude-redshift samples complete in apparent magnitude. They proposed a robust test for independence, free of assumption concerning the spatial distribution of sources, which allows to estimate the cosmological parameters characterizing the geometry of the Universe from a quasar sample (see also Efron \& Petrosian 1999). It turns out that this statistical test, given a world model, can easily be recycled for testing the completeness assumption. The purpose of the present paper is exactly to take advantage of such a possibility.

The statistical background of the method as well as the test for completeness are presented section 2. An example of application is given in section 3, where the test is used for investigating the completeness in apparent magnitude of the South Sky Redshift Survey of da Costa et al. (1998). The properties of the new completeness test and its range of application are finally summarised in section 4 .

\section{THE COMPLETENESS TEST}

\subsection{Assumptions and statistical model}

The luminosity function of the galaxies population is herein defined following Bingelli et al. (1988) as the probability distribution function $f_{t}(M)$ of the absolute magnitude $M$ of the galaxies depending in general on the epoch $t$. At any epoch, the luminosity function is by definition normalised (i.e. $\int f_{t}(M) d M=1$ ). It is assumed hereafter that the luminosity function of the population does not depend on the $3 \mathrm{D}$ redshift space position $\mathbf{z}=(z, l, b)$ of the galaxies. Without accounting for selection effects in observation, the probability density describing the population splits under this assumption as

$d P_{\mathbf{z} M} \propto d P_{\mathbf{z}} \times d P_{M}=\rho(z, l, b) d l d b d z \times f(M) d M$

where $\rho(z, l, b)$ is the $3 \mathrm{D}$ redshift space distribution function of the sources along the past light-cone.

The present model is thus well suited to describe the observed spatial fluctuations of the galaxies density and to account for a pure density evolution scenario (i.e. the variations of the mean galaxies density with redshift or equivalently with time). On the other hand, Eq. (伍) fails to describe environmental effects (i.e. the luminosity function of the sampled objects depends on the local environment) and an evolution of the specific characteristics (e.g. mean absolute magnitude, shape) of the luminosity function.

Selection effects in observation enter the statistical model as a filter response function (see for example Bigot\&Triay 1990). This selection function $\psi$ can be expressed in general in terms of the observable quantities, namely herein the line-of-sight direction $(l, b)$, the redshift $z$ and the raw apparent magnitude $m$, i.e. $\psi \equiv \psi(m, z, l, b)$. Accounting for selection effects in observation, the probability density describing the sample may be written as

$d P=\frac{1}{A} \psi(m, z, l, b) \rho(z, l, b) f(M) d l d b d z d M$

with $A$ the normalisation factor warranting $\int d P=1$.
The null hypothesis tested hereafter is that the sample is complete in raw apparent magnitude up to a given magnitude limit $m_{\text {lim }}$, or in other words where the selection function in apparent magnitude is well described by a sharp cut-off, i.e.

$\mathrm{H} 0: \psi(m, z, l, b) \equiv \theta\left(m_{\mathrm{lim}}-m\right) \times \phi(z, l, b)$

with $\theta(x)$ the Heaviside or 'step' function. The function $\phi(z, l, b)$ describes some eventual selection effects in angular position and observed redshift. For example, it could account for a mask in angular position as well as pure selection or subsampling in redshift (e.g. a lower and upper limits).

The absolute magnitude $M$ is obtained following

$M=m_{\text {cor }}-\mu(z)$

where the distance modulus $\mu(z)$ can be evaluated from the redshift given a cosmological world model $\left\{H_{o}, \Omega_{o}, \Lambda_{o}\right\}$ (see for example Weinberg 1972). Note that it has been implicitly assumed herein that the contribution of peculiar velocities to the observed redshifts is negligible. The corrected apparent magnitude $m_{\text {cor }}$ is expressed as

$m_{\text {cor }}=m-k_{\text {cor }}(z)-A_{g}(l, b)$

with $k_{\text {cor }}(z)$ standing for a k-correction term and $A_{g}(l, b)$ accounting for a Galactic extinction correction.

At this stage, it is convenient to introduce the quantity $Z$ defined as

$Z=m-M=\mu(z)+k_{\mathrm{cor}}(z)+A_{g}(l, b)$

which can be computed from the observables $z$ and $(l, b)$ providing a world model. Under the null hypothesis of Eq. (3), i.e. the sample is complete in apparent magnitude, the probability density of Eq. (2) may be rewritten using these notations as

$d P=\frac{1}{A} h(Z, l, b) d l d b d Z f(M) d M \theta\left(m_{\lim }-m\right)$

where the distribution function $h(Z, l, b)$ may be expressed, if required, in function of the $3 \mathrm{D}$ redshift space distribution $\rho(z, l, b)$, the selection function $\phi(z, l, b)$ introduced Eq. (3) and using the definition of $Z$ given Eq. (6). The cut-off in apparent magnitude introduces a correlation between the variables $M$ and $Z$ (intrinsically faint and distant galaxies are discarded, see figure 1) wich would have been statistically independent otherwise.

\subsection{The random variable $\zeta$}

Thanks to the introduction of the quantity $Z$, the maximum absolute magnitude $M_{\lim }(Z)$ for which a galaxy at a given $Z$ would be visible in the sample is uniquely defined, i.e.

$M_{\lim }(Z)=m_{\lim }-Z$

The milestone of the method consists in defining the random variable $\zeta$ as follows

$\zeta=\frac{F(M)}{F\left(M_{\lim }(Z)\right)}$

where $F(M)$ stands for the Cumulative Luminosity Function, i.e.

$F(M)=\int_{-\infty}^{M} f(x) d x$ 


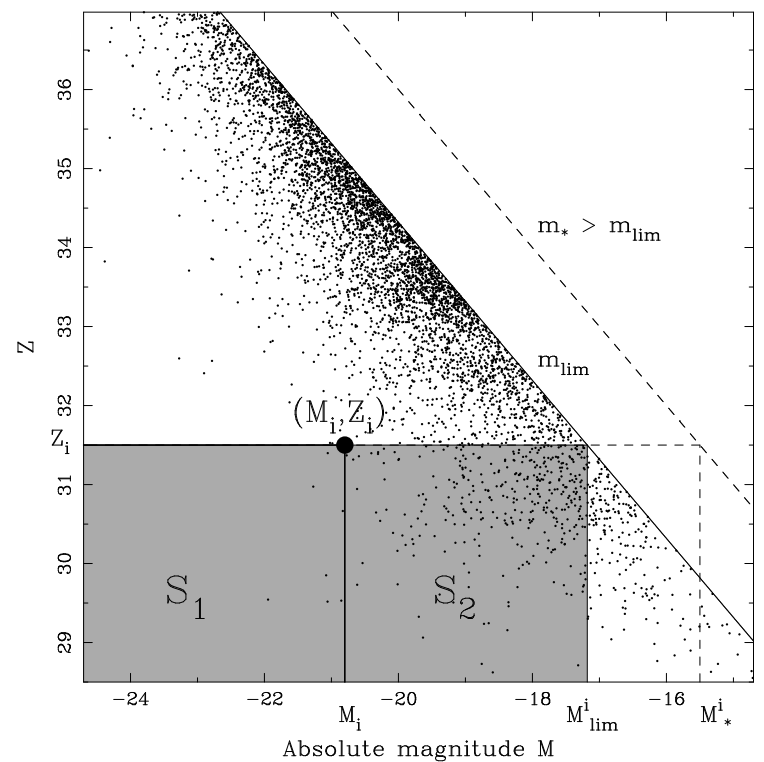

Figure 1. The variable $Z$ defined Eq. (6) versus absolute magnitude $M$. The procedure for evaluating $r_{i}$ and $n_{i}$ entering the calculation of the random variable $\hat{\zeta}_{i}$ of Eq. (15) is illustrated.

The volume element of Eq. (8) may thus be rewritten as

$d l d b d Z d \zeta=\frac{f(M)}{F\left(M_{\lim }(Z)\right)} d l d b d Z d M$

and by definition the random variable $\zeta$ for a sampled galaxy belongs to the interval [0,1]. The probability density of Eq. (đ) reduces therefore to

$d P=\frac{1}{A} h(Z, l, b) F\left(M_{\lim }(Z)\right) d l d b d Z \times \theta(\zeta) \theta(1-\zeta) d \zeta$

with $A=\int h(Z, l, b) F\left(M_{\text {lim }}(Z)\right) d l d b d Z$. It follows from Eq. (12) that:

- P1: $\zeta$ is uniformly distributed between 0 and 1 .

- P2: $\zeta$ and $(Z, l, b)$ are statistically independent.

Property P1 will be used hereafter to construct the test for completeness.

\subsubsection{Estimate of the random variable $\zeta$}

Under the null hypothesis $\mathrm{H} 0$, the random variable $\zeta$ can be estimated without any prior knowledge of the cumulative luminosity function $F(M)$. Let us consider the distribution of the sampled galaxies in the $M-Z$ diagram (see figure 1 ). To each point with coordinates $\left(M_{i}, Z_{i}\right)$ is associated the region $S_{i}=S_{1} \cup S_{2}$ defined as

- $S_{1}=\left\{(M, Z)\right.$ such that $M \leq M_{i}$ and $\left.Z \leq Z_{i}\right\}$

- $S_{2}=\left\{(M, Z)\right.$ such that $M_{i}<M \leq M_{\mathrm{lim}}^{i}$ and $\left.Z \leq Z_{i}\right\}$

The random variables $M$ and $Z$ are independent in each subsample $S_{i}$ since by construction the cut-off in apparent magnitude is superseded by the constraints $M<M_{\lim }^{i}\left(Z_{i}\right)$ and $Z \leq Z_{i}$ (see figure 1). It implies from Eq. (đ) that the number of points $r_{i}$ belonging to $S_{1}$ is given by

$\frac{r_{i}}{N_{\text {gal }}}=F\left(M_{i}\right) \times \frac{1}{A} \int_{-\infty}^{Z_{i}} h(Z, l, b) d Z d l d b$ with $N_{\text {gal }}$ the number of galaxies in the sample, and that the number of points $n_{i}$ in $S_{i}=S_{1} \cup S_{2}$ is

$\frac{n_{i}}{N_{\text {gal }}}=F\left(M_{\lim }\left(Z_{i}\right)\right) \times \frac{1}{A} \int_{-\infty}^{Z_{i}} h(Z, l, b) d Z d l d b$

The numbers $r_{i}$ and $n_{i}$ are obtained by merely counting the galaxies respectively belonging to $S_{1}$ and $S_{1} \cup S_{2}$. An unbiased estimate of the random variable $\zeta$ introduced Eq. (9) is indeed provided by the quantity

$\hat{\zeta}_{i}=\frac{r_{i}}{n_{i}+1}$

Using rank-based statistics, Efron \& Petrosian (1992) prove moreover that the random variables $\hat{\zeta}_{i}$ are independent of each other under H0. The expectation $E_{i}$ and variance $V_{i}$ of the $\hat{\zeta}_{i}$ are respectively

$E_{i}=\frac{1}{2} \quad ; \quad V_{i}=\frac{1}{12} \frac{n_{i}-1}{n_{i}+1}$

Note that the value of the variance $V_{i}$ tends towards the variance of a continuous uniform distribution between 0 and 1 when $n_{i}$ becomes large enough.

It turns out that the quantity $T_{C}$ defined as

$T_{C}=\sum_{i=1}^{N_{\text {gal }}}\left(\hat{\zeta}_{i}-\frac{1}{2}\right) /\left(\sum_{i=1}^{N_{\text {gal }}} V_{i}\right)^{\frac{1}{2}}$

has an expectation zero and variance unity under $\mathrm{H} 0$. The statistic $T_{C}$ proposed herein is almost similar to the Efron \& Petrosian test statistic for independence based on the normalised ranks.

The $T_{C}$ statistic can be estimated without assuming any prior model for the luminosity function. It is worthwhile to mention that no assumptions have been made as well concerning the distribution function $h(Z, l, b)$ introduced Eq. (1). It means that the property of the $T_{C}$ statistic derived above holds for any 3D redshift space distribution $\rho(z, l, b)$ (allowing the presence of clustering and the evolution of the mean galaxies density with time), and for any selection function $\phi(z, l, b)$ (e.g. subsampling in redshift bins would not bias the $T_{C}$ statistic).

\subsection{The test for completeness $T_{C}$}

The principle of the test is to evaluate the quantity $T_{C}$ defined Eq. (17) for subsamples truncated to increasing apparent magnitude limit $m_{*}$, i.e. $m_{*}$ is replacing $m_{\lim }$ in Eq. (8).

As long as $m_{*}$ remains below the completeness limit $m_{\text {lim }}$ the subsample is obviously complete up to $m_{*}$ and the $T_{C}$ statistic is thus expected to be distributed around zero with sampling fluctuations of dispersion of unity. On the other hand as $m_{*}$ becomes greater than $m_{\text {lim }}$, the incompleteness introduces a deficit of galaxies with $M$ fainter than $M_{\text {lim }}(Z)$ (see figure 1). It results in a lack of galaxies with a value of $\hat{\zeta}_{i}$ close to unity. Figure 2 dramatically illustrates such a trend for a sample characterized by a strict cut-off in $m_{\text {lim }}$, but the trend would remain similar for a smooth imcompleteness function as well. It turns out that the $T_{C}$ statistic is expected to be systematically negative for limiting apparent magnitude $m_{*}$ greater than the completeness limit $m_{\text {lim }}$. 


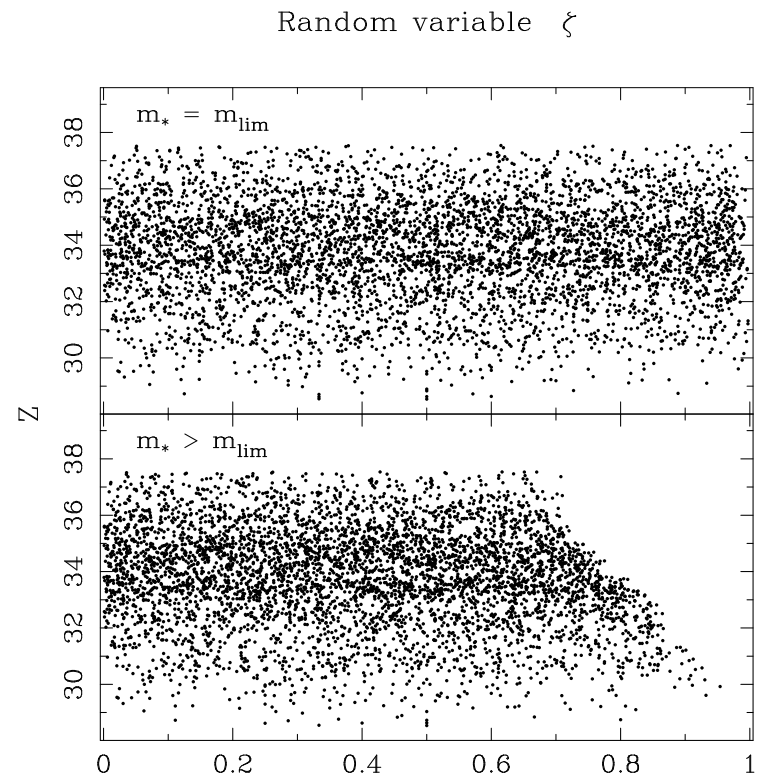

Figure 2. Diagram $\zeta-Z$ for two values of the limiting apparent magnitude $m_{*}$. For $m_{*}$ greater than the completeness limit $m_{\text {lim }}$, the number of galaxies fainter than $M_{\lim }(Z)$ is underestimated due to incompleteness (see figure 1), inferring a systematic lack of points with a value of $\hat{\zeta}_{i}$ close to unity. This effect is particularly visible at high $Z$ (i.e. distant galaxies).

Therefore, the curve $T_{C}\left(m_{*}\right)$ is characterized by a plateau of zero mean for $m_{*}$ below $m_{\text {lim }}$, followed by a systematic decline beyond this apparent magnitude, i.e.

$T_{C} \simeq 0$ for $m_{*} \leq m_{\lim } ; T_{C}<0$ for $m_{*}>m_{\lim }$

Under H0, the sampling fluctuations make the $T_{C}$ statistic to follow a gaussian distribution of variance one. The decision rule for fixing the completeness limit is a matter of choice, having in mind that the confidence levels of rejection associated to the events $T_{C}<-1, T_{C}<-2, T_{C}<-3$ are respectively $84.13 \%, 97.72 \%, 99.38 \%$ (i.e. these numbers correspond to the probabilities drawn from a Normal distribution for a one-sided rejection test).

\section{EXAMPLE OF APPLICATION}

The test for completeness $T_{C}$ is herein applied to the South Sky Redshift Survey (SSRS2) sample of da Costa et al. (1998). The sample, containing 5369 galaxies with measured B-band magnitude and redshift, has been drawn primarily from the list of nonstellar objects identified in the Hubble Space Telescope Guide Star Catalog (Lasker et al. 1990). The redshift survey is more than $99 \%$ complete up to the magnitude limit $m_{\mathrm{SSRS} 2}$ of $15.5 \mathrm{mag}$ (da Costa et al. 1998). The test for completeness $T_{C}$ is thus applied to assess the completeness in apparent magnitude of the primary list of galaxies used to build on the SSSR2 sample.

The type-dependent k-correction are calculated following Pence (1976), i.e. $k_{\text {cor }}(z)=K_{B}(T) \times c z /\left(10000 \mathrm{~km} \mathrm{~s}^{-1}\right)$ with $K_{B}(T)=0.15$ for $T \leq 0, K_{B}(T)=0.15-0.025 T$ for $3 \geq T \geq 0$ and $K_{B}(T)=0.075-0.010(T-3)$ for $3 \leq T$. Galactic extinctions are obtained as $A_{g}(l, b)=$ $4.325 E(B-V)$ by use of the dust maps of Schlegel et al. (1998) for the redenning correction.
The redshifts have been transformed in the CMB rest frame and the distance modulus is computed adopting an Hubble constant of $H_{0}=100 \mathrm{~km} \mathrm{~s}^{-1} \mathrm{Mpc}^{-1}$ in a flat universe with no cosmological constant (i.e. $\Omega_{0}=1$ and $\left.\Lambda_{0}=0\right)$.

Galaxies not belonging to the redshifts range $[2500,15000] \mathrm{km} \mathrm{s}^{-1}$ are discarded. The lower limit in redshift has been introduced in order to minimize the impact of peculiar velocities on the $T_{C}$ statistic. In particular, the kinematical influence of the Virgo cluster will be considerably reduced by removing nearby galaxies. The upper bound in redshift sets some limits on the interval of time spanned by the data and thus reduces the influence of an eventual evolution of the luminosity function on the $T_{C}$ statistic $(c z=15000$ $\mathrm{km} \mathrm{s}^{-1}$ corresponds to a look-back time of $0.510^{9}$ years for $H_{0}=100 \mathrm{~km} \mathrm{~s}^{-1} \mathrm{Mpc}^{-1}$, respectively 1 Gyr if $H_{0}=50 \mathrm{~km}$ $\mathrm{s}^{-1} \mathrm{Mpc}^{-1}$ ). Note that such a subsampling in redshift is not expected to affect the result of the search.

Finally, a random component uniformly distributed between $[-0.005,0.005]$ has been added to the catalogued magnitudes (the apparent magnitudes of the SSRS2 sample are rounded to $0.01 \mathrm{mag}$ ). Rounding problems have negligible effects on the present analysis but can infer spurious variations of the $T_{C}$ statistic. Because magnitudes are set to discrete values, it creates some artificial gaps in the magnitude distribution function. The effect is observable on datasets rounded high and containing a large number of galaxies, e.g. the Zwicky catalogue (Zwicky et al. 1968).

The test is applied to subsamples truncated to increasing values of the limiting apparent magnitude $m_{*}$. For each galaxies with coordinates $\left(M_{i}, Z_{i}\right)$, the quantity $M_{\lim }\left(Z_{i}\right)=$ $m_{*}-Z_{i}$ is formed, the numbers $r_{i}$ and $n_{i}$ are computed, as well as the random variable $\hat{\zeta}_{i}$ and its variance $V_{i}$. The statistic $T_{c}\left(m_{*}\right)$ is obtained following Eq. (17) by summing over all the galaxies of the subsample. The results are shown figure 3 , bottom panel. Considering the value of $T_{C} \approx-5$ at $m_{*}=15.5$, it is clear that the completeness in apparent magnitude of the SSRS2 sample is not satisfied up to the magnitude 15.5. Or in other words, the assumption that the sample is complete up to 15.5 is rejected at a confidence level greater than $5 \sigma$. The rule for deciding which value of the completeness limit has to be adopted is on the other hand a matter of choice. Herein a $2 \sigma$ criterion has been chosen to reject the completeness hypothesis, leading to a value of $m_{\text {lim }}=15.35$ for the completeness in apparent magnitude.

The decimal logarithm of the number count versus the limiting apparent magnitude is shown figure 3, top panel. A slope of 0.6 is expected if galaxies were uniformly distributed in space (i.e. this is the standard completeness test proposed in Hubble 1926). The test does not allow to draw any firm conclusions concerning the completeness of the sample. The observed slope appears to be slightly shallower than 0.6 but nothing prevents the effect to be due to inhomegeneity in the spatial distribution of the galaxies. Moreover, no particular trend is visible beyond the $m_{*}=15.35$ limit where the $T_{C}$ statistic indicates that the SSRS2 sample suffers from incompleteness.

The application of the $T_{C}$ statistic to the SSRS2 sample allows to conclude, with a high confidence level, that the sample is not complete in apparent magnitude up to $m_{\mathrm{SSRS} 2}=15.5 \mathrm{mag}$. However, the significant deviation of the $T_{C}$ statistic from zero may be due to hidden systematic 


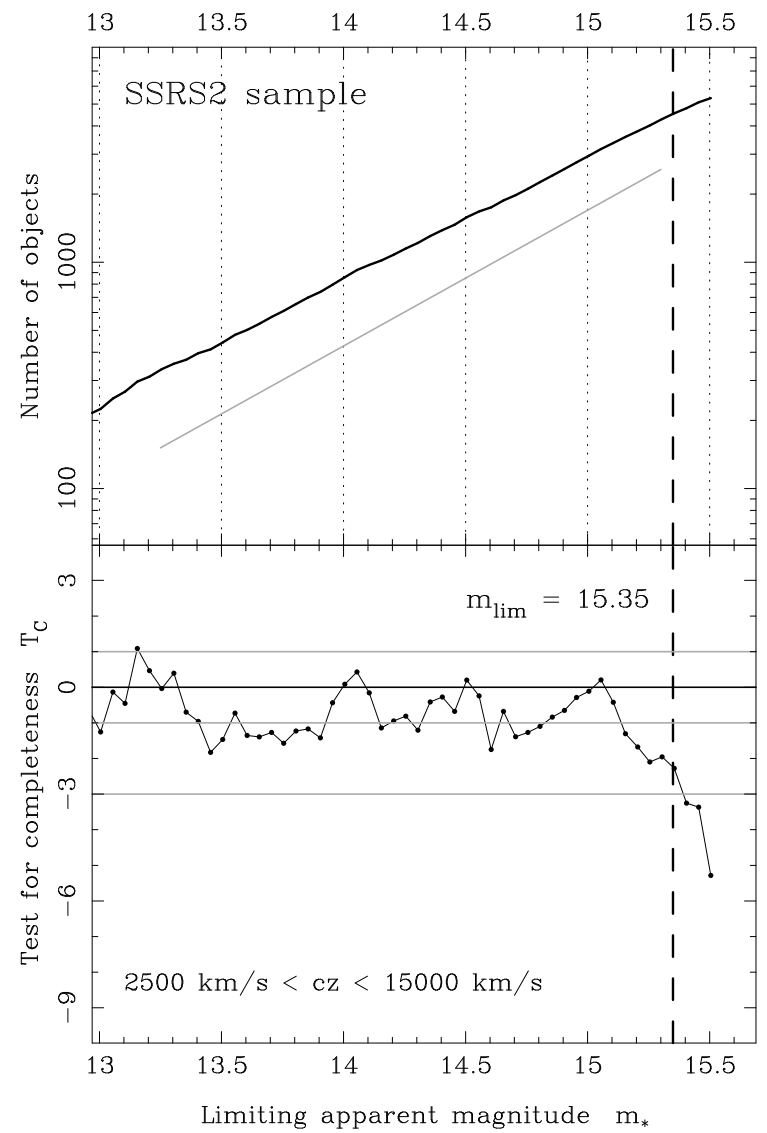

Figure 3. The test for completeness $T_{C}$ applied to the the 4324 galaxies (all types) of the SSRS2 sample with redshifts between 2500 and $15000 \mathrm{~km} \mathrm{~s}^{-1}$. A systematic decline of the $T_{C}$ statistics can be observed beyond $m_{\lim }=15.35$. Top panel shows in logarithmic scale the galaxies number count in function of the limiting apparent magnitude $m_{*}$. The 0.6 slope (gray line) is the slope expected if the sources were uniformly distributed in space.

effects. In particular, it has been assumed that the luminosity function does not depend on the spatial position of the galaxies. It is well known that the E/SO galaxies, in contrast to spirals, populate preferentially galaxy clusters (see for example Loveday et al. 1995). If the luminosity functions of E/SO and spiral galaxies are indeed different (say for example that $\mathrm{E} / \mathrm{SO}$ galaxies are brighter in average), the luminosity function of the whole population (E/SO+spirals) is expected to depend on the spatial position (in average, galaxies will be brighter in clusters than in the field). Such an environmental effect could influence the $T_{C}$ statistic and therefore affect the conclusions drawn from the completeness test. The influence of E/S0 and spiral galaxies segregation is investigated figure 4 . The completeness test has been applied separately to the two populations. The systematic decline of the $T_{C}$ statistic can be observed for both types and the completeness limit of $m_{\mathrm{SSRS} 2}=15.5 \mathrm{mag}$ is ruled out with a high confidence level of rejection $(\approx 4 \sigma)$.

As pointed out by Jon Loveday, the referee of this paper, other potential problems may affect the present analysis. It has been assumed for example that the k-correction and the Galactic extinction map used herein are correct. An improper correction of these quantities could lead to systematic biases for the $T_{C}$ statistic. The influence of such

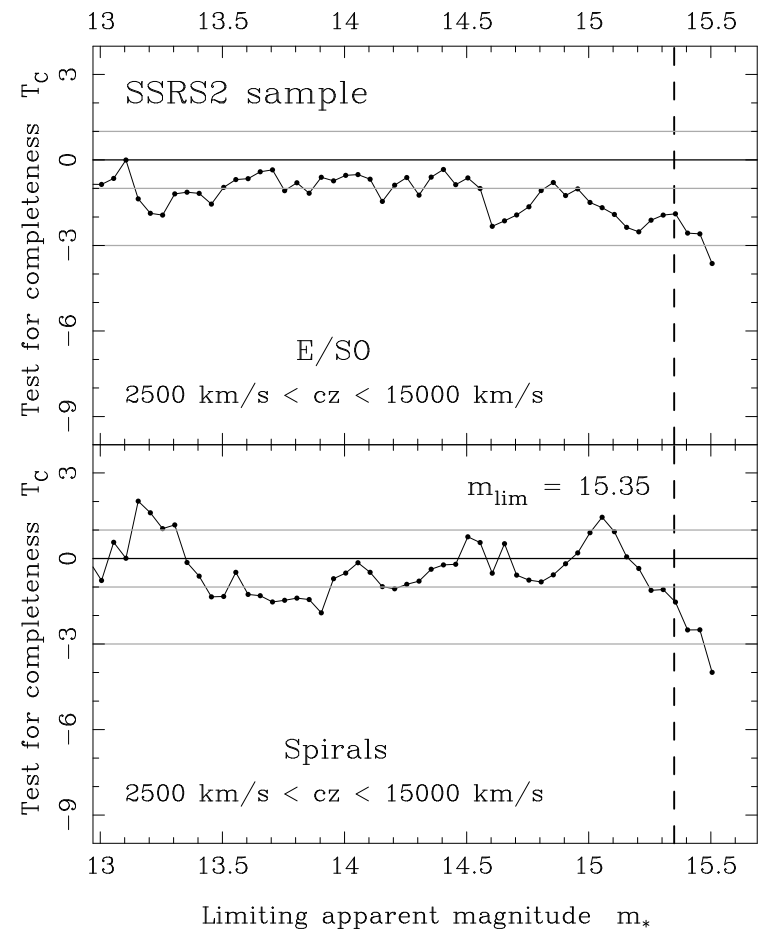

Figure 4. The test for completeness $T_{C}$ applied to the $1373 \mathrm{E} / \mathrm{SO}$ galaxies (Top) and to the 2780 spirals galaxies (Bottom) of the SSRS2 sample with redshifts between 2500 and $15000 \mathrm{~km} \mathrm{~s}^{-1}$. The systematic decline at $m_{\mathrm{lim}}=15.35$ is present for both E/SO and spirals galaxies.

effects is investigated in figure 5. The top panel shows the completeness test applied to a subsample of all types nearby galaxies of the SSRS2 survey $\left(2500 \mathrm{~km} \mathrm{~s}^{-1}<c z<7500 \mathrm{~km}\right.$ $\mathrm{s}^{-1}$ ). The k-correction for this subsample is therefore small, $0.048 \mathrm{mag}$ in average with a maximum of $0.11 \mathrm{mag}$. The decline of the $T_{C}$ statistic at $m_{\lim }=15.35$ is still present, suggesting that an improper k-correction term cannot explain the imcompleteness observed in figure 3 . The bottom panel of figure 5 shows the completeness test applied to the all types SSRS2 galaxies with a Galactic extinction $A_{g}(l, b)$ lesser than 0.1 mag. Note that such a subsampling is not supposed to bias the $T_{C}$ statistic since selection effect in angular direction are allowed (see section 2.2.1). Again, the $T_{C}$ statistic falls beyond $m_{\lim }=15.35$. It thus appears that the systematic deviation of the completeness test is not due to an eventual improper correction for the k-correction or for the Galactic extinction.

Another potential problem is that the k-correction is herein type dependent, which is not described by the statistical formalism presented section 2.1, at least if the luminosity function is also type dependent. To be strictly valid, the completeness test has to be applied type by type. Six subsamples of spirals have been selected (from type $T=1$ to $T=6)$. The result of the completeness test at $m_{*}=15.5$ mag on these subsamples is respectively $T_{C}=-1.51,-1.09$, $-2.73,-2.33,-0.94$ and $T_{C}=-1.6$, which indicates that in average the completeness of the sample is not satisfied up to $m_{\lim }=15.5 \mathrm{mag}$. Note however that the incompleteness is less flagrant in this type-by-type analysis than for the all spirals subsample of figure $4\left(T_{C}=-4\right.$ at $m_{*}=15.5$ mag). It is not surprising since, as any rejection test, the 


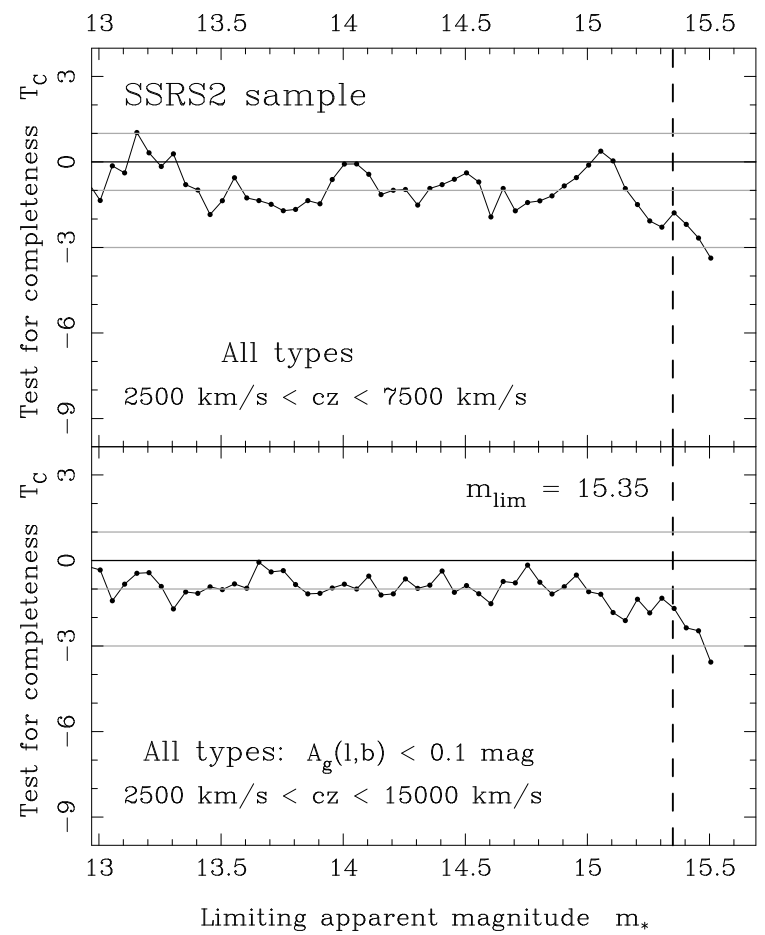

Figure 5. The test for completeness $T_{C}$ applied to the SSRS2 all types galaxies (top) with redshifts between 2500 and $7500 \mathrm{~km} \mathrm{~s}^{-1}$ (2416 galaxies with a mean k-correction of $0.048 \mathrm{mag}$ ) (bottom) with redshifts between 2500 and $15000 \mathrm{~km} \mathrm{~s}^{-1}$ and a galactic extinction $A_{g}(l, b)<0.1 \mathrm{mag}$ (1260 galaxies). The systematic decline at $m_{\text {lim }}=15.35$ is present in both cases, suggesting that the imcompleteness cannot be explained by an improper k-correction term or Galactic extinction correction.

efficiency of the $T_{C}$ statistic is improved as the number of the datapoints increases.

The results of the test for completeness on the SSRS2 sample can be summarised as follows. The test for completeness indicates that the primary list of galaxies used to build on the SSRS2 redshifts sample is not complete in appparent magnitude to the $m_{\mathrm{SSRS} 2}=15.5 \mathrm{mag}$ limit. Rather, it suggests to adopt $m_{\mathrm{SSRS} 2}=15.35 \mathrm{mag}$ (or less) as a reasonable completeness limit. The point is of importance since incompleteness in apparent magnitude is source of biases when evaluating the intrinsic characteristics of the galaxies luminosity function for example (Marzke et al. 1998). A special attention has been paid to investigate the systematic effects which could bias the $T_{C}$ statistic (influence of peculiar velocities, evolution of the luminosity function with time, environmental effect, influences of the k-correction and galactic extinction).

\section{SUMMARY}

A new tool has been proposed for assessing the completeness in apparent magnitude of a magnitude-redshift sample. The technique presents a real improvement compared to standard completeness tests. Namely, no a priori assumptions are required concerning the redshift space distribution of the sources. It means in particular that neither the clustering nor the evolution of the mean number density of the galaxies do affect the result of the search.
The test for completeness presented herein is however reliable if and only if the magnitude-redshift sample verifies the following criteria:

1) The distances of the galaxies are required, which implies that a cosmological world model has to be specified and that the contribution of peculiar velocities to observed redshifts can be safely neglected. Furthermore, the galactic extinction and the k-correction involved in the definition of the absolute magnitudes are required.

11) The shape of the luminosity function of the galaxies is not allowed to change with time (in practice, this criterion is achieved if the sample is divided into thin intervals of redshift, i.e. time).

111) The luminosity function of the population is assumed to be independent on the spatial position of the galaxies. In particular, environmental effect may affect the results of the completeness test.

\section{ACKNOWLEDGEMENTS}

I would like to thank Richard Barrett, Martin Hendry, Gilles Theureau and David Valls-Gabaud for fruitful discussions. I acknowledges the support of the PPARC and the use of the STARLINK computer node at Glasgow University.

\section{REFERENCES}

Bigot G., Triay R., 1990, Phys. Lett. A, 150, 227

Binggeli B., Sandage A., Tammann G. A., 1988, ARA\&A, 26, 509

Branchini E., Teodoro L., Frenck C. S., Schmoldt I., Efstathiou G., White S. D. M., Saunders W., Sutherland W., RowanRobinson M., Keeble O., Tadros H., Maddox S., Oliver S. 1999, MNRAS 308, 1

da Costa L. N., Willmer C. N. A., Pellegrini P. S., Chaves O. L., Rité C., Maia M. A. G., Geller M. J., Latham D. W., Kurtz M. J., Huchra J. P., Ramella M., Fairall A. P., Smith C., Lípari S., 1998, AJ, 116, 1

Driver S. M., 1999, ApJ Letters, 526, L69

Efron B., Petrosian V., 1992, ApJ, 399, 345

Efron B.. Petrosian V., 1999, J. Amer. Statist. Assoc., 447, 824 astro-ph/9808334)

Heavens A. F., Taylor A. N., 1997, MNRAS, 290, 456

Hubble E., 1926, ApJ, 64, 321

Hudson M. J., Lynden-Bell D., 1991, MNRAS, 252, 219

Lasker B. M., Sturch C. R., McLean B. M., Russel J. L., Jenker H., Shara M., 1990, AJ, 99, 2019

Loveday J., Maddox S. J., Efstathiou G., Peterson B. A., 1995, ApJ, 442, 457

Lynden-Bell D., 1971, MNRAS, 155, 95

Marzke R. O., da Costa L. N., Pellegrini P. S., Willmer N. A., Geller M. J., 1998, ApJ, 503, 617

Pence W., 1976, ApJ, 203, 39

Petrosian V., 1976, ApJ Letters, 209, L1

Rauzy S., Hendry M. A., 2000, MNRAS, 316, 621

Sandage A., Tammann G. A., Yahil A., 1979, ApJ, 232, 352

Sandage A., Perelmuter J.-M., 1990, ApJ, 350, 481

Schlegel D. J., Finkbeiner D. P., Davis M., 1998, ApJ, 500, 525

Schimdt M., 1968, AJ, 151, 393

Wienberg S., 1972, Gravitation and Cosmology, J. Wiley, NewYork

Zwicky F., Herzog E., Wild P., Karpowicz M., Kowal C. T., 19611968, Catalogue of Galaxies and of Clusters of Galaxies, Vols 1-6 (Pasadena: California Institute of Technology) 